\title{
SARS CoV-2 adenovirus and RNA based vaccines potential autoimmune complications: could we lower the chances?
}

\author{
Mina Kelleni ${ }^{1}$ \\ ${ }^{1}$ Affiliation not available
}

August 2, 2021

\begin{abstract}
Nucleic acid based - mRNA based and adenovirus vectored - vaccines, were first ever or first commercially ever approved for the public, respectively. However, these newly emergency approved types possess a potential risk to induce auto-immune diseases e.g., thrombocytopenia, myocarditis and immune induced thrombosis and thromboembolism that might be fatal and could reason for some of the post vaccination sudden death reports. Moreover, all SARS CoV-2 types of vaccines, depending on the spike protein immunogenicity, especially the conventional inactivated ones might increase the likelihood of COVID-19 severity upon re-infection through antibody dependent enhancement which might reason for the recently described abundance of hospital admissions within seven days of vaccination and might also reason for some of the serious adverse effects encountered with administration of convalescent plasma to COVID-19 patients as well as they might share in development of some lethal SARS CoV-2 variants. Importantly, we suggest that SARS CoV-2 mass vaccination campaigns were the worst ever decision made and that making these COVID-19 vaccines compulsory or administering them to children or pregnant participants might be considered as a crime against humanity to the extent that no prior companies- governmental agreements would ever secure impunity. Finally, a full informed personalized risk benefit ratio especially for some described high-risk groups must be secured while suggesting that the subunit vaccines are the least hazardous ones.
\end{abstract}

\section{Hosted file}

SARS CoV-2 Vaccines Complications1.pdf available at https://authorea.com/users/318758/ articles/496102-sars-cov-2-adenovirus-and-rna-based-vaccines-potential-autoimmunecomplications-could-we-lower-the-chances

\section{Hosted file}

SARS CoV-2 Vaccines Complications.pdf available at https://authorea.com/users/318758/ articles/496102-sars-cov-2-adenovirus-and-rna-based-vaccines-potential-autoimmunecomplications-could-we-lower-the-chances

Two clinical trials of SARS CoV-2 adenovirus vector -based vaccines have been temporarily halted due to autoimmune complications concerns in some participants. Similarly, SARS CoV-2 RNA vaccines possess a theoretical potential to elicit autoimmune complications. After their approval to combat the current COVID19 pandemic, nucleic acid-based vaccination, whether DNA or RNA, is considered first of its kind to be used in humans. Potential risks including development of autoimmune diseases might only be documented when used on a large scale as the clinical trials have involved only tens of thousands of participants. Thus, a strict system for post marketing surveillance must be secured to report any potential adverse effect and the techniques used in development of these types of vaccines should focus on innovative methods to decrease their potential autoimmunity. Importantly, smokers, obese and diabetic participants are more liable groups to develop autoimmune diseases and we recommend a personalized risk benefit ratio to be evaluated before vaccination 
waiting for further safety data coming from post marketing surveillance. Finally, quitting smoking, loss of overweight and control of blood glucose levels might help to lower their probabilities in case of the benefits exceeded the risks.

Short communication

SARS CoV-2 adenovirus vector and RNA based vaccines potential autoimmune complications: A Reasonable Explanation for the Sudden Death Reports.

Mina T. Kelleni, MD, PhD

pharmacology Department, College of Medicine, Minia University, Egypt

mina.kelleni@mu.edu.eg; drthabetpharm@yahoo.com

Mobile: +201200382422

https://orcid.org/0000-0001-6290-6025

Key messages:

Adenovirus vector and RNA based SARS CoV-2 vaccines possess a potential risk to induce auto-immune diseases.

Nucleic acid-based vaccination is considered first of its kind to be used in humans after their approval in Russia and UK to combat COVID-19

Clinical trials involved only tens of thousands of participants and potential autoimmune complications might be revealed only post marketing.

Autoimmune myocarditis might be the reason for the cardiac arrest/sudden death reports encountered during the clinical trials and post marketing (I've updated the text after the report coming from Portugal, sent to another journal and today we read about the report of the Florida Physician who died of immune thrombocytopenia complications, I wish I might have time soonest to update also the text here).

Smokers, obese and diabetic individuals are in general more liable to develop auto-immune diseases and an individualized risk benefit ratio should be considered before vaccination.

An advice to quit smoking, lose overweight and control of blood glucose level might prove beneficial to lower the incidence of these potential autoimmune complications.

Keywords: COVID-19, SARS CoV-2, adenovirus vector vaccines, mRNA vaccines, autoimmune diseases.

Graphical abstract 


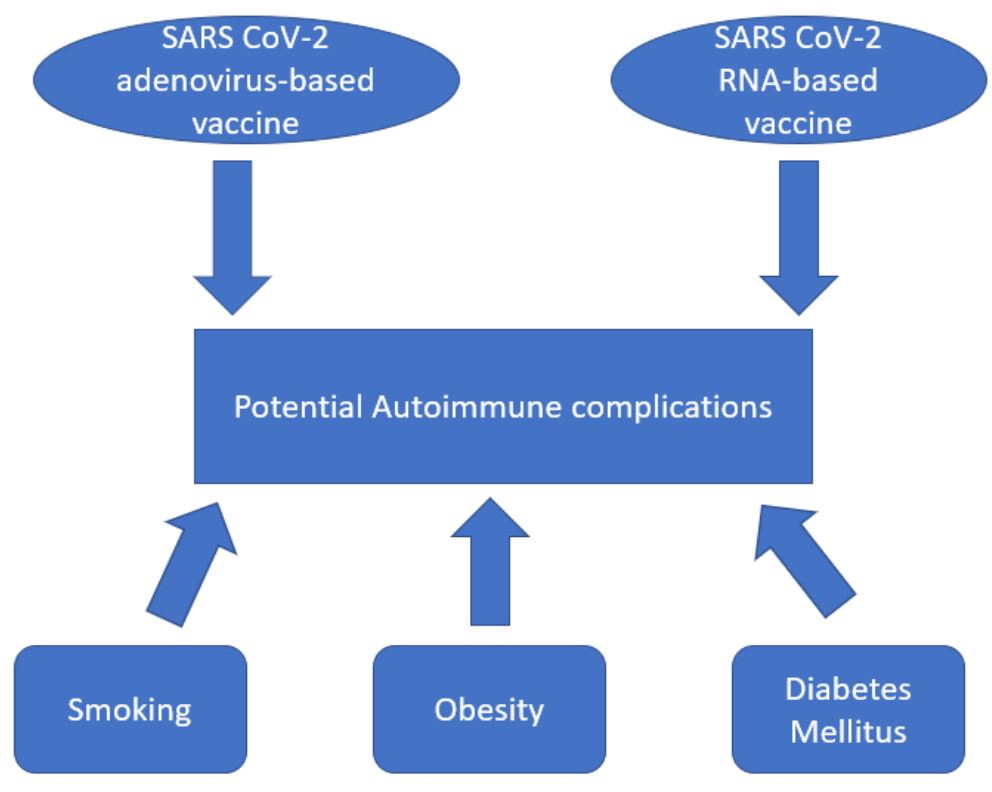

Smokers, obese individuals and diabetic patients are considered more vulnerable to develop autoimmune diseases which are also considered as potential adverse effects of SARS CoV-2 adenovirus and RNA-based vaccines.

\section{Introduction}

COVID-19 vaccines are considered of utmost importance to stem SARS CoV-2 current pandemic, with 48 candidates in clinical evaluation, including seven adenovirus, five messenger RNA (mRNA) and one selfamplifying RNA based vaccines encoding spike protein of SARS CoV-2 as declared by the WHO on the $12^{\text {th }}$ of November 2020[1,2]. However, the unprecedent accelerated timelines to develop some of COVID-19 vaccines have necessitated a call for active pre and post-licensure safety surveillance systems to properly investigate potential adverse effects or toxicities [1,3]. Importantly, two adenovirus vector-based SARS CoV-2 vaccine global phase III clinical trials were temporarily paused due to reports of serious adverse medical events of autoimmune complications including multiple sclerosis and transverse myelitis which were ultimately deemed to be unrelated to the SARS CoV-2 vaccine. However, lack of transparency concerns have been raised as the involved companies declined the release of the thorough details of these serious adverse events claiming privacy issues [4-7] and a sharp criticism of the analysis of the results of one trial including a serious dose mistake that involved thousands of patients has also been raised[8]. Notably, adenovirus vector SARS CoV-2 vaccine has been first commercially approved in humans in Russia and is undergoing a mass vaccination program $[9,10]$.

\section{RNA based vaccines potential hazards}

On the other hand, RNA based vaccines, recently first approved in UK for COVID-19 as a first ever approval for this novel type of vaccination in a western country [11], possess multiple theoretical and manufacturing advantages over traditional subunit, live attenuated and killed virus vaccines[12-14]. However, in practice the results of two previous clinical trials using mRNA vaccines to prevent H10N8, H7N9 influenza viruses and rabies have been lower than what was expected when compared to those of their preclinical studies[13]. Similarly, though mRNA vaccines encoding HIV and CMV antigens elicited antigen-specific CD4+ and CD8+ T cell immune responses; no reduction in viral load was observed[12]. 
Similar to adenovirus vector-based vaccines, potential risks of mRNA and saRNA based vaccines also include risk of potential autoimmunity with possible development of autoreactive antibodies of any non-native nucleotides and delivery system components $[13,15,16]$. All SARS CoV-2 adenovirus vector and RNA vaccines phase III clinical trials involved only tens of thousands of patients and this risk cannot be fully excluded until post marketing safety data prove their safety. Notably, soon after its mRNA based SARS CoV-2 vaccine approval, the UK Medicines and Healthcare products Regulatory Agency advised people with a history of significant allergic reactions to medicines, food or vaccines not to have the jab [17] and the identification of individuals at an increased risk of autoimmune reactions before mRNA vaccination was advised[13]. We would like to explore some groups of individuals who are potentially more vulnerable to autoimmune diseases, aiming to recommend a personalized risk benefit ratio to be considered before a decision to be immunized by adenovirus and RNA based SARS CoV-2 vaccine until encouraging post marketing safety data are revealed for all SARS CoV-2 types of vaccines.

Who are the higher-risk groups, and could we lower their chances to develop autoimmune diseases?

The first higher-risk group are smokers; cigarette smoke has been reported to lead to an enhanced risk of inflammatory and autoimmune diseases[18]. Smokers are more likely to develop critical COVID-19 requiring mechanical ventilation [19] that might lead to a higher mortality rate [20, 21]. Interestingly, alarms about the danger of misreading non-significant or inconclusive frequentist results containing several possible biases of a contradictory hypotheses have been raised [22, 23].

Two other important groups that might be closely monitored include obese and diabetic individuals; obesity was suggested to be a major environmental factor contributing to the onset and progression of autoimmune diseases[24] and a concomitant autoimmune disease was encountered as 1 in 4 of 179,248 people diagnosed with type 1 diabetes[25]. Notably, a meta-analysis has showed diabetes, but not obesity, to be linked to a higher COVID-19 mortality[26]. However, increasing risks of COVID-19 hospital death were noticed to be associated with increasing levels of obesity (BMI $>40$ fully adjusted HR 2.27, 95\% CI 1.99-2.58)[27].

Interestingly, quitting smoking at diagnosis was recently shown to decrease the risk of death in cancer patients[28], and quitting smoking was suggested to alleviate its impact in patients with pneumonia and other COVID-19 associated infections[21, 23, 29], thus a beneficial advice to quit smoking together with another to lose overweight and to control the blood glucose levels might also help to lower the chances of SARS $\mathrm{CoV}-2$ adenovirus and RNA-based vaccine potential autoimmunity in those individuals. Most importantly, we would like to stress the utmost importance to remind the participants to report all experienced adverse effects to a well-prepared post marketing surveillance system. Further, the search to improve methods that help to develop nucleic acid based vaccines with minimal autoimmune potential risk should continue and until more reassuring post marketing safety data are released, we recommend considering an individualized risk benefit ratio especially for those higher risk groups of patients.

Conflict of interests

the author declares he has no conflict of interest

Funding/Financial disclosure

None

References:

[1] Kochhar S, Salmon DA. Planning for COVID-19 vaccines safety surveillance. Vaccine 2020;38(40):6194-8.

[2] World Health Organization. Draft landscape of COVID-19 candidate vaccines. 2020, November 12 .

[3] Petousis-Harris H. Assessing the Safety of COVID-19 Vaccines: A Primer. Drug Safety 2020. 
[4] Reuters (Ludwig Burger). AstraZeneca pauses coronavirus vaccine trial as participant illness investigated, https://www.reuters.com/article/us-health-coronavirus-astrazeneca/astrazenecapauses-coronavirus-vaccine-trial-rollout-doubts-dent-shares-idUSKBN26017L; 2020, September 09.

[5] Statnews ( MATTHEW HERPER). Covid-19 vaccine trials from AstraZeneca, Johnson \& Johnson to restart, https://www. statnews. com/2020/10/23/covid-19-vaccine-trials-from-astrazenecajohnson-johnson-to-restart/; 2020, October 23.

[6] Ledford SMH. COVID-vaccine results are on the way - and scientists' concerns are growing. Nature. 2020, September 25.

[7] Reuters. J\&J pauses COVID-19 vaccine trials due to unexplained illness in participant, https://www.reuters.com/article/uk-health-coronavirus-johnson-johnson/jj-pausescovid-19-vaccine-trials-due-to-unexplained-illness-in-participant-idUKKBN26Y02L; 2020, October 13 .

[8] The New York Times (Rebecca Robbins and Benjamin Mueller). After Admitting Mistake, AstraZeneca Faces Difficult Questions About Its Vaccine, https://www.nytimes .com/2020/11/25/business/ coronavirus-vaccine-astrazeneca-oxford.html; 2020, November 25.

[9] ABC News. Russia starts mass coronavirus vaccination in Moscow with its Sputnik V shot, https://www . abc.net.au/news/2020-12-05/russia-starts-mass-covid-19-vaccinationmoscow/12954380; 2020, December 5.

[10] Ryan Cross. Adenoviral vectors are the new COVID-19 vaccine front-runners. Can they overcome their checkered past? CHEMICAL \& ENGINEERING NEWS,

. 98. 2020, May 12 .

[11] BBC (Michelle Roberts). Covid-19: Pfizer/BioNTech vaccine judged safe for use in UK from next week, https://www.bbc.com/news/health-55145696; 2020, December 02.

[12] Zhang C, Maruggi G, Shan H, Li J. Advances in mRNA Vaccines for Infectious Diseases. Frontiers in Immunology 2019;10(594).

[13] Pardi N, Hogan MJ, Porter FW, Weissman D. mRNA vaccines - a new era in vaccinology. Nature Reviews Drug Discovery 2018;17(4):261-79.

[14] McKay PF, Hu K, Blakney AK, Samnuan K, Brown JC, Penn R, et al. Self-amplifying RNA SARS-CoV-2 lipid nanoparticle vaccine candidate induces high neutralizing antibody titers in mice. Nature communications 2020;11(1):3523-.

[15] Wang F, Kream RM, Stefano GB. An Evidence Based Perspective on mRNA-SARS-CoV-2 Vaccine Development. Med Sci Monit 2020;26:e924700-e.

[16] Bloom K, van den Berg F, Arbuthnot P. Self-amplifying RNA vaccines for infectious diseases. Gene Therapy 2020.

[17] BBC News (Nick Triggle and Rachel Schraer). Covid-19 vaccine: Allergy warning over new jab, https://www.bbc.com/news/health-55244122; 2020, December 09.

[18] Hussain MS, Tripathi V. Smoking under hypoxic conditions: a potent environmental risk factor for inflammatory and autoimmune diseases. Mil Med Res 2018;5(1):11.

[19] Adrish M, Chilimuri S, Mantri N, Sun H, Zahid M, Gongati S, et al. Association of smoking status with outcomes in hospitalised patients with COVID-19. BMJ Open Respir Res 2020;7(1). 
[20] Jimenez-Ruiz CA, Lopez-Padilla D, Alonso-Arroyo A, Aleixandre-Benavent R, Solano-Reina S, de Granda-Orive JI. COVID-19 and Smoking: A Systematic Review and Meta-Analysis of the Evidence. Arch Bronconeumol 2020.

[21] Patanavanich R, Glantz SA. Smoking is associated with worse outcomes of COVID-19 particularly among younger adults: A systematic review and meta-analysis. medRxiv 2020.

[22] Usman MS, Siddiqi TJ, Khan MS, Patel UK, Shahid I, Ahmed J, et al. Is there a smoker's paradox in COVID-19? BMJ Evidence-Based Medicine 2020:bmjebm-2020-111492.

[23] Carmona-Bayonas A, Jimenez-Fonseca P, Sánchez Arraez Á, Álvarez Manceñido F, Castañón E. Does active smoking worsen Covid-19? Eur J Intern Med 2020;77:129-31.

[24] Versini M, Jeandel PY, Rosenthal E, Shoenfeld Y. Obesity in autoimmune diseases: not a passive bystander. Autoimmun Rev 2014;13(9):981-1000.

[25] Rogers MAM, Wei MY, Kim C, Lee JM. Sex Differences in Autoimmune Multimorbidity in Type 1 Diabetes Mellitus and the Risk of Cardiovascular and Renal Disease: A Longitudinal Study in the United States, 2001-2017. J Womens Health (Larchmt) 2020;29(4):511-9.

[26] Figliozzi S, Masci PG, Ahmadi N, Tondi L, Koutli E, Aimo A, et al. Predictors of adverse prognosis in COVID-19: A systematic review and meta-analysis. European Journal of Clinical Investigation 2020;50(10):e13362 .

[27] Williamson E, Walker AJ, Bhaskaran KJ, Bacon S, Bates C, Morton CE, et al. OpenSAFELY: factors associated with COVID-19-related hospital death in the linked electronic health records of 17 million adult NHS patients. medRxiv 2020:2020.05.06.20092999.

[28] Yang S, Liu T, Liang G. The benefits of smoking cessation on survival in cancer patients by integrative analysis of multi-omics data. Mol Oncol 2020.

[29] CDC. Coronavirus Disease 2019 (COVID-19): People with Certain Medical Conditions, https://www.cdc.gov/coronavirus/2019-ncov/need-extra-precautions/people-with-medicalconditions.html\#smoking; 2020, November 02. 